\title{
Homelessness and companion animals: more than just a pet?
}

\author{
Jessica Slatter, ${ }^{1}$ Chris Lloyd ${ }^{2}$ and Robert King ${ }^{3}$
}

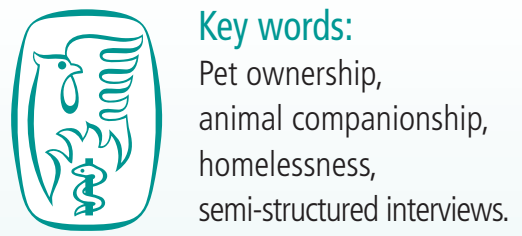

${ }^{1}$ Occupational Therapist, Evolve Therapeutic Services, Gold Coast Health Service District, Southport, Queensland, Australia.

2 Principal Research Fellow, Gold Coast Health Service District, and Senior Research Fellow, Behavioural Basis of Health, Griffith University, Gold Coast Campus, Southport, Queensland, Australia. 3 Professor, School of Psychology and Counselling, Queensland University of Technology, Kelvin Grove, Queensland, Australia.

Corresponding author: Dr Chris Lloyd, Senior Research Fellow, Behavioural Basis of Health, Griffith University, Gold Coast Campus, Southport, Queensland 4215, Australia. Email: c.lloyd@griffith.edu.au

Reference: Slatter J, Lloyd C, King R (2012) Homelessness and companion animals: more than just a pet? British Journal of Occupational Therapy, 75(8), 377-383.

\section{DOI: 10.4276/030802212X13433105374350}

(C) The College of Occupational Therapists Ltd. Submitted: 2 November 2011

Accepted: 17 February 2012.
Purpose: Research identifies the value of animal companionship for people of various ages and with physical and mental health diagnoses. However, there is limited research into the value of animal companionship for the homeless population.

Method: This exploratory study aimed to investigate the value that homeless people find in animal companionship; the extent to which homelessness impacts on the ability to have animal companions; and interventions that health professionals can implement to reduce barriers to animal companionship. Twenty-six people were interviewed during Homeless Health Outreach Team outreach. Descriptive analysis of the responses to the semi-structured interviews was carried out.

Findings: Results indicated that the majority of participants had given up a pet, and wanted a pet but could not due to their homelessness. The impact of giving up a companion animal was significant. Difficulties associated with pet ownership focused mostly on the living environment and the cost. There were several benefits to having companion animals. Participants viewed that having a pet made a difference to their lives in that it provided friendship and responsibility and contributed to emotional wellbeing.

Conclusion: It is suggested that occupational therapists need to be aware of the impact that pet ownership has on the lives of homeless people and to explore ways in which they can assist with this.

\section{Introduction}

Homeless Health Outreach Teams (HHOTs) have been established across the state of Queensland, Australia, to improve the mental health outcomes of homeless individuals. HHOTs are multidisciplinary, and incorporate a mix of allied health clinicians, mental health practitioners and drug and alcohol specialists. Service delivery is based on recovery, assertive outreach and partnership building models. Primary and secondary homelessness are included in eligibility criteria. The cultural definition of homelessness used by HHOTs comes from Chamberlain and MacKenzie (1992):

1. Primary homelessness (rough sleepers; people without conventional accommodation, including improvised dwellings)

2. Secondary homelessness (couch surfers, people who move frequently from one form of temporary shelter to the next)

3. Tertiary homelessness (medium to long term boarding house residents). This article describes the themes from the literature concerning animal companions. It then goes on to describe a small research project that was conducted to examine the value that homeless people find in animal companionship.

\section{Themes from the literature review}

\section{Terminology: pet versus animal companion}

Many dictionaries define pets as domesticated animals that are kept for companionship. Significant relationships are formed with these animals as they live, 
work and play with their human families on a daily basis (Zimalag and Krupa 2009). Having a pet is a part of Australian culture and a familiar concept worldwide. More recently, animal welfare groups and veterinary professionals have adopted the term 'companion animal' to describe pets. The new phrase aims to reflect better the mutual psychological bond between person and animal (Walsh 2009).

\section{Recent pet owner trends}

A national United States (US) survey conducted during 2007-2008 found that 63\% of American homes had a pet; 95\% of pet owners regarded their pet as their friend; $87 \%$ regarded their pet as family; $53 \%$ of pet owners took time off work for a sick animal; 44\% took their pets to work with them; and $87 \%$ included their pet in holiday celebrations (Walsh 2009). The Australian Companion Animal Council (ACAC 2009) found that the same number of Australian homes had a pet in 2007; more than 83\% of Australians had a pet at some time in their lives; and 53\% of those without a pet wanted one in future. Ninety-one per cent of pet owners felt very close to their pet and $81 \%$ never felt alone when with their pet.

\section{Family and attachment theories: comfort and companionship}

Phillips Cohen (2002) conducted a study involving forced choice questions to examine what is meant when people say their pet is a member of the family. Results indicated that animal companions were definitely inside the family circle; however, they were not perceived as being human family. More participants felt that they could rely on the emotional support and comfort from their pet than from their human family members. Phillips Cohen (2002) stressed that animal companions occupy an overlapping but different space in the family, and having a strong bond with an animal did not imply a lack of close human relationships.

Animal related activities tend to carry symbolic meaning, including nurturing, holding, talking, feeding and caring (Velde et al 2005). When a person and animal live their lives together, it is natural for individuals to experience profound grief at the loss of their animal companion, which can be as strong as for a human (Phillips Cohen 2002, O'Haire 2009, Walsh 2009). Attachment theories have been expanded to understand the relationships that people have with animal companions (Zimalag and Krupa 2009). Family systems theory also provides a framework for understanding the various roles that pets have in families (Walsh 2009).

\section{Animals as social lubricants}

Companion animals have been described as social icebreakers and social lubricants for their human partners by various researchers. Studies have indicated that when people walk their dogs, they experience significantly more unplanned encounters than without a dog being present (McNicholas and Collis 2000, Collins et al 2006). People are also more likely to interact with neighbours and participate in community activities when they have companion animals (ACAC 2009,
Wood 2009). Adults who used wheelchairs were found to be more frequently smiled at and spoken to when accompanied by their service dogs than when travelling alone (Collins et al 2006).

Research studies have explored the concept of pets as social enablers, and found that the presence of animals can positively and unconsciously modify opinions of others. A study demonstrated this using pairs of identical pictures, with the only difference being an animal present in one of the identical pairs. When animals were in the image, it was described as a more pleasant and less fear-provoking scene by participants (O'Haire 2009).

\section{Barriers to animal companionship: accommodation and finances}

Australians are increasingly experiencing barriers to animal companionship. The trend has been toward urban and highdensity living, which often results in expensive or apartment accommodation (ACAC 2009, O'Haire 2009, Zimalag and Krupa 2009).

A study conducted in the US by Shore et al (2003) found that the most frequently given reason for pet relinquishment was the owner relocating. Often the new accommodation had policies that did not permit animals or was selective about the type, size or number of animals allowed. People were required to move for various reasons, including employment changes, rental conditions, financial difficulties or other life transitions (Shore et al 2003).

Renters and low-income earners have been described as the population most at risk of animal relinquishment, given the limited options available when seeking new accommodation (Brown and Rhodes 2006). Pet-friendly rentals are becoming out of reach for Australians, with many rental policies prohibiting animals. Just $17 \%$ of rental properties have pets in Australia compared with the national average of two out of three homes (ACAC 2009). Rental agreements are more tolerant in Ontario, Canada, where the Residential Tenancies Protection Act only permits pet owners to be evicted if the animal affects the wellbeing of other tenants (Zimalag and Krupa 2009).

\section{Animal companionship and physical health}

Research has suggested that pets protect against stress-related physical health problems. Interactions with pets can increase neurochemicals associated with relationships and bonding and, therefore, improve immune system functioning (Walsh 2009). Animals have been effectively used in preoperative settings to reduce anxiety, improve positive attitude and reduce the need for medication (Velde et al 2005).

Longitudinal studies from Germany and Australia have shown that animal companionship results in fewer doctor visits, and those who had a companion animal were healthier than those who no longer or never had one (McNicholas and Collis 2000). There is some evidence to show that pet ownership could result in lowered cardiovascular levels and that animal companions buffer autonomic responses to acute stress, which may explain the health outcomes observed in these 
studies (Virués-Ortega and Buela-Casal 2006). The benefits are mutual: patting a companion dog reduces blood pressure in both the person and his or her animal (Walsh 2009).

\section{Animal companionship and mental health}

Animal companions can play a role in the psychological health of humans by reducing stress, anxiety, depression and loneliness (Le Roux and Kemp 2009). Velde et al (2005) conducted a study into animal assisted therapy with individuals who had severe mental illness. The occupational therapists found that participants showed improved motivation, sensory modulation, attention and concentration, social interaction, responsibility and emotional wellbeing

It is frequently documented that people with serious mental illness experience social and occupational marginalisation. The contribution to health and wellbeing that animals bring to people with a range of mental illnesses has been documented for half a century. Despite the long history of companionship between animals and people, Wood (2009) described the relationship being undervalued and negatively diagnosed by mental health professionals.

De Souza (2000) found "people with mental illness felt that pets provided an opportunity to care for "someone" without the complexities of human relationship dynamics which are often dependent on conditional love and approval' (cited in Zimalag and Krupa 2009, p128). Enders-Slegers (2000) indicated that 'being responsible for the well-being of another living being enhances feelings of self-worth and self esteem (cited in Zimalag and Krupa 2009, p128). Zimalag and Krupa (2009) stated that given the social and emotional benefits of having companion animals, it is important to note that a minority of those with serious mental illness in the community have a pet.

\section{Animal companionship and homelessness}

Homelessness has often been described as causing significant social exclusion: 'Homelessness is a state in which people are not only excluded from basic shelter, but also from security, a place to belong, intimate relationships, neighbours, and all of the benefits of having a place to call one's own' (Talbot 2003, cited in Noble-Carr 2006, p38).

Families accounted for $26 \%$ of the Australian homeless population in 2006 (Gibson and Johnstone 2010). Families experiencing violence are particularly vulnerable to homelessness. Women and children who enter crisis accommodation often have difficulties obtaining stable housing due to the limited affordable accommodation available (Lee 2008). Family pets are also often threatened and harmed in violent homes, and 33\% of women have reported delaying leaving a violent relationship due to concern for the welfare of pets, as many women's refuges do not permit animals (Lee 2008).

Children experiencing homelessness face stressors including the loss of home, schooling, friendships, pets, and extended family members (Lee 2008). Children commonly report missing pets and belongings when homeless, with almost half of children surveyed in one study expressing the loss of pets as their greatest concern (Keys 2009).
Rew's (2000) research identified that more than a third of homeless adolescents found value in having a dog. The need to care for an animal appeared to encourage responsibility and good decision making. The animal also provided the opportunity for unconditional love and emotional development. Rew (2000) concluded that companion animals might make the difference between just surviving on the streets and integrating back into the community.

\section{Method}

\section{Study design}

Ethics approval for this study was gained from the Gold Coast Health Service District Ethics Committee. The overall aim was to investigate the experience of homeless people with respect to pet ownership. More specifically, the authors wanted to find out how important pets are to homeless people and how homelessness impacts upon pet ownership. The study took the form of a cross-sectional investigation, using semi-structured interviews with people receiving outreach support from clinicians of the Gold Coast HHOT.

\section{Sample}

The sample consisted of 26 participants. Nineteen males and seven females took part in the study. Their ages ranged between 20 and 60 years. All but four people were recruited from free food venues, with the remaining interviews completed at emergency accommodation shelters.

\section{Semi-structured questionnaire}

A panel of people with expertise in developing questions for research met to create two separate semi-structured questionnaires. One was designed for those who presently had an animal companion, and the other was for those who did not. Each contained approximately 10 questions, including:

- What is your history of involvement with pets?

- What barriers have you encountered because of having pets?

- What do you value about having a pet?

- Have you had to give away a pet because of your living circumstances; if yes, how did this affect you?

- Would you like to have a pet now and, if yes, what are the main things stopping you?

\section{Procedure}

The principal researcher selected a convenience sample during HHOT outreach: every fourth person queuing up for food. Prospective participants were provided with an information sheet, which was read aloud to them. They were also given the option of keeping a copy of this. If individuals accepted the invitation to participate in the research, they were requested sign a consent form. Interviews were conducted predominantly by the principal researcher. These were all face to face and lasted approximately 45 minutes. The interviews were not audio recorded; however, the researcher transcribed the responses as they took place. 


\section{Data analysis}

Consensual qualitative research (CQR) (Hill et al 1997) was the means used for data analysis in this study. Specifically, CQR highlights the use of multiple researchers, the process of reaching consensus and a systematic way of examining the representativeness of results across cases (Hill et al 1997). The CQR process involves three general steps, which are:

- Responses to open-ended questions from questionnaires or interviews for each individual case are divided into domains (that is topic areas)

- Core ideas (that is abstracts or brief summaries) are constructed for all the material within each domain for each individual case

- A cross analysis, which involves developing categories to describe consistencies in the core ideas within domains across cases, is conducted (Hill et al 1997).

The consensus process is central to the CQR method. A team approach was used to extract themes and to classify the responses to each of the questions. This involved the principal researcher and the two other researchers in, first, reviewing all responses and identifying themes. They then met as a group to present and discuss their ideas until they reached a single version that all team members endorsed. The strength of a team approach is that it enhances the reliability of classification of themes, reducing risks associated with a single coder.

Quotations that were illustrative of specific themes were identified.

\section{Findings}

Almost a quarter of the participants had a pet at the time of interview, with the majority of these having a dog. Duration of pet ownership was between 1 and 10 years. Almost twothirds of participants stated that they would like to have a pet if it were possible, with the majority preferring a dog.

\section{Theme 1: Grief and loss}

The majority of participants told stories of having lost animal companions at some time during their lives, as a direct result of being homeless. For many, it was after being evicted and not being able to take the animal to sleep rough on the streets or into the next accommodation facility, while for several others companion animals were lost following difficulties meeting council regulations:

I was living somewhere and got a kitten. I got kicked out, and had to give it away for a while. When I tried to get it back I was told it had been put down.

The pound took my dog due to someone complaining about it. I couldn't afford to get it back.

While rough sleeping I had a dog and the council took it off me and put it down. It wasn't registered, and I couldn't afford to get it back.

There was a strong theme of grief and loss, with participants explaining, 'I was sad. It was like losing a friend', 'I was really attached to the cat, it was my best friend. I was sad and the cat was distressed' and 'it's like a part of me is taken away'.

Participants also spoke of their guilt, 'I didn't know if I had done the right thing', and feeling as though they had no control over their life. Others spoke of their anger, loneliness, and decreased emotional wellbeing.

\section{Theme 2: Barriers to caring for pets while homeless}

The main difficulties participants had experienced in the past were the cost of keeping pets and the lack of appropriate living conditions. Difficulties accessing public transport and meeting council regulations were also factors discussed by several participants:

I couldn't find affordable accommodation. It was a choice between being homeless or giving up the cat.

One participant stated that he had experienced fewer opportunities of getting accommodation because of policies preventing pets, 'I would go without myself, it's hard getting accommodation'. One person who did not identify barriers to having pets in the past said, 'I wouldn't have any difficulties if I wasn't homeless'. A third of the participants currently wanted a pet, but felt this was not possible due to their homelessness. Half of participants identified accommodation policies preventing pets as being a reason for not being able to have a pet. This included public housing, 'I'm in public housing so am not allowed to have one', as well as temporary accommodation, 'Not allowed to have one if going into crisis accommodation'.

Those who were seeking private rental accommodation also anticipated barriers: 'if there's no backyard then I have nowhere to keep it' and 'I just got a studio apartment, and am not allowed to have one. It should be a human right to own an animal'.

For those participants who were sleeping rough, they explained: 'it's not possible, I have nowhere to keep it', 'I'm not settled, not secure' and 'I'm unemployed, I can't afford food and vet bills'.

\section{Theme 3: Pets as a source of emotional resilience}

Participants were given the opportunity to reflect on the various ways that having a pet did or would make a difference to their life. More than two-thirds of participants explained how companion animals provided company, companionship and friendship. One participant explained, 'I love having pets around, they make you good, you hug and kiss them, sleep with them, watch movies together, laugh'. Another reflected similar thoughts, stating his pet provided valuable benefits: 'friendship, always having someone to talk to who won't argue and will agree on everything'

Pets appeared to mitigate a sense of isolation from meaningful relationships with others, with two participants explaining their pets give 'company, as I'm on my own' and 'non judgmental affection, someone who wants to be with me'. A number of participants also found that they experienced 
increased social interaction from having a pet. One spoke of how his pet 'puts me in touch with other people' while another said more 'friends like to hang around'.

More than a third of participants experienced 'more responsibility, which is a good thing'. The increased sense of responsibility appeared to provide other benefits as well, including increased motivation, organisation and routine, as explained by two participants:

I would be happy, have something to do, a reason to get up, as I would have to feed it

I would be less lonely, more responsible, more organised, cause you have to feed it. I would have a routine.

Emotional wellbeing was another theme identified, with over a quarter of participants describing improved mental health as being a benefit of animal companionship. Pets 'would make me a bit more happy, animals keep me healthy'. Pets 'keep my mind off my problems'. Another participant experienced an increased self-awareness of emotion regulation, explaining:

You have to curb yourself. If you wake up in a bad mood, you have to check yourself. It's like having children.

Physical health and activity were not as frequently discussed by participants. Physical health and emotional health were often discussed together:

We walk twice a day. I'm in touch with my emotions. It's better than blood pressure medicine, with him, I'll live longer.

... they get me out walking, get me motivated, boost my emotions, give me more confidence, be more relaxed. I'm sad that I can't have one.

Interestingly, but surprisingly, only a few of the participants found the protection that their dog gave them while sleeping rough was beneficial: 'Night can be dangerous on the streets. She will warn you if someone is coming. My mates all love her too' and 'They look after you, you look after them. It's easier to sleep and relax and feel more at ease when protected'.

\section{Discussion}

Clinical experience with the HHOT highlighted that homeless people face many barriers to maintaining their relationship with animal companions. It appeared that they commonly face the dilemma of whether to choose accommodation over homelessness to keep their pets.

When compared with the overall Australian population, those who are homeless do not differ considerably in their experiences of animal companionship. The ACAC (2009) found that approximately two-thirds of Australian households have pets. This study has found that an equal number of homeless individuals wanted to have a pet but felt that it was not possible due to their homelessness, and only one quarter currently had a pet. The ACAC (2009) also found that $83 \%$ of Australians had a pet at some time in their lives.
A similar figure of $76 \%$ participants in this study had lost an animal at some point of their lives due to being or becoming homeless. It could be suggested that, in Australia, having and keeping a pet is limited to those who can afford it.

Unfortunately, many of the people in this study had to give up their pet because of significant environmental challenges. It was found by Shore et al (2003) that landlords play a crucial role in the need to relinquish a pet when changing residence. They found that a combination of relatively low income and status as renters suggested that pet owners might have relatively few options other than to relinquish the animal.

Both the literature review and the findings from this study have identified that animal companionship contributes to the health and wellbeing of people who are homeless in tangible ways. It is evident that the participants interviewed for this study have experienced a range of benefits from their companion animal relationships. Two consistent findings have been that value of companion animals in engaging people who are homeless in meaningful occupations; and the improved emotional wellbeing through the experience of companionship. A participant in this study summed this up in a sentence: 'Contentment, responsibility, cheers me up, and cuddles'. The Allen et al (2000) study echoed this, finding that animal companionship involved two dimensions: emotional attachment and the structure of daily routines and responsibilities.

In looking at homeless youth, Rew (2000) found that caring for a dog assisted some of the participants to cope with homelessness in ways that enabled them not merely to survive but also to develop in healthy ways. Having a socially valued occupation can enable people to build capacity, productivity and community participation (Zimalag and Krupa 2009). It could be assumed that animal companionship, as an occupation for homeless individuals, may be an opportunity to build the skills required to reintegrate into mainstream society. Despite these connections, it seems that there has been a lack of occupational therapy literature about the link between animal companionship and the impact on daily occupations of adults living in the community (Zimalag and Krupa 2009).

Many studies have highlighted companionship and unconditional love as significant reasons for pet ownership (Allen et al 2000, Zimalag and Krupa 2009). Over half of the participants in this study also identified this as their reason for wanting a companion animal. The emotional benefits found in this study have been reflected in numerous other studies and include decreased loneliness, increased self-esteem and increased social interactions.

\section{Treatment implications and recommendations}

Marginalisation and a lack of productive and meaningful roles are challenges for individuals with serious mental illness, and the benefit of companion animals with this population is an area that has not received much attention in occupational therapy (Zimalag and Krupa 2009). It seems particularly important that occupational therapists explore the value of animal companions even more when individuals are occupationally and socially isolated. 
Animal companionship requires cognitive, social and emotional skills, and can provide structuring of daily routines, responsibilities and quality use of time (Allen et al 2000, Zimalag and Krupa 2009). Given that occupational therapy aims to promote engagement in meaningful occupations to improve quality of life, it is fitting that the profession further investigates its role in promoting animal companionship (Zimalag and Krupa 2009).

Occupational therapists can assist individuals to establish and maintain animal relationships through person, occupation and environment focused interventions (Zimalag and Krupa 2009). Person-level interventions might include cognitive skills training, such as following schedules and problem solving. Occupation-level interventions may involve grading responsibilities or activities, starting with low maintenance animals or tasks. Environment-level interventions would consider the physical and social setting when choosing type of animal, linking individuals with community services and supports, and teaching individual rights and responsibilities with tenant agreements (Zimalag and Krupa 2009).

Human-animal bonds merit greater attention in mental health research, and in family systems theory and practice. There is increasing evidence that pets provide important physiological and relational benefits, and contribute to wellbeing and healing and to positive growth. Humananimal bonds are almost unmentioned in mental health training programmes, despite the abundance of literature (Zimalag and Krupa 2009).

Health professionals in the clinical setting can quickly and simply measure the strength of human-animal bonds. Researchers indicated that clinicians could enquire whether a picture is carried of companion animals or if they were considered as members of the family, in order to measure bonds (Shore et al 2003). Asking how much time is spent with their pet may also assist health professionals to assess relationships with companion animals, and those who spent more than 16 hours per day together were more likely to have particularly strong attachments (Phillips Cohen 2002).

Recommendations specific to HHOT staff may include allowing clinicians to take a dog with them on outreach. This may assist in facilitating interactions with homeless individuals and in establishing rapport. Interventions that assist homeless people to engage in volunteer work with animal welfare agencies, or to gain the skills necessary to take care of a pet of their own, are also recommended. Occupational therapists may also develop further roles in advocating for homeless individuals and those with mental illness to obtain pet-friendly housing.

\section{Limitations of the study}

A cross-sectional study does not provide a basis for causal inferences. The evidence for a direct causal association between human wellbeing and companion animals is not conclusive; however, the literature is largely supportive of the widely held and long-standing belief that pets are good for us. This study is limited further by the fact that it took place in one geographical area and consisted of only a small sample size. The consistency of findings of this study and those previously reported provides some basis for confidence that the findings are more widely generalisable, but caution is nonetheless required.

\section{Conclusion}

This was an exploratory study, designed to investigate the experiences of animal companionship for individuals who are homeless. The study involved semi-structured, crosssectional interviews with participants from the Gold Coast region. It was found that homeless people valued having an animal because of companionship, a sense of responsibility, emotional wellbeing and the increased social activity associated with caring for a pet. The main barriers to maintaining pets were housing policies, costs and lack of suitable facilities to support pet ownership.

\section{Acknowledgements}

We would like to thank the participants who gave their time to this project. Conflict of interest: None declared.

\section{Key findings}

- Pet ownership appeared to be a valued occupation.

- The issue of grief and loss over having lost a pet was dominant.

- Pets were seen as a source of emotional resilience.

What the study has added

This study has increased occupational therapists' awareness of the important role that pets can play in homeless people's lives.

\section{References}

Allen JM, Hammon Kellegrew D, Jaffe D (2000) The experience of pet ownership as a meaningful occupation. Canadian Journal of Occupational Therapy, 67(4), 271-78.

Australian Companion Animal Council (2009) The power of pets: the benefits

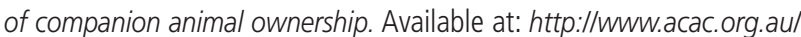
pdf/PowerOfPets_2009_19.pdf Accessed 09.04.11.

Brown SG, Rhodes RE (2006) Relationship among dog ownership and leisuretime walking in western Canadian adults. American Journal of Preventative Medicine, 30(2), 131-36.

Chamberlain C, MacKenzie D (1992) Understanding contemporary homelessness: issues of definition and meaning. Australian Journal of Social Issues, 27(4), 274-97. Cited in: C Gibson, T Johnstone (2010) Investing in our future: children's journeys through homelessness and child protection. A scan of the literature, policy and practice. Adelaide: Australian Centre for Child Protection, University of South Australia.

Collins DM, Fitzgerald SG, Sachs-Ericsson N, Scherer M, Cooper R, Boninger ML (2006) Psychosocial well-being and community participation of service dog partners. Disability and Rehabilitation: Assistive Technology, 1(1-2), 41-48.

De Souza HR (2000) Use of the Delphi technique and qualitative method to compare the way in which mental health clients and providers understand the meaning of quality of life. Unpublished Master's thesis. Kingston, ON: Queen's University. Cited in: U Zimalag, T Krupa (2009) Pet ownership as a meaningful community occupation for people with serious mental illness. American Journal of Occupational Therapy, 63(2), 126-37. 
Enders-Slegers MJ (2000) The meaning of companion animals: qualitative analysis of the life histories of elderly cat and dog owners. In: AL Podberscek, ES Paul, JA Serpell, eds. Companion animals and us. Cambridge: Cambridge University Press, 237-56. Cited in: U Zimalag, T Krupa (2009) Pet ownership as a meaningful community occupation for people with serious mental illness. American Journal of Occupational Therapy, 63(2), 126-37.

Gibson C, Johnstone T (2010) Investing in our future: children's journeys through homelessness and child protection. A scan of the literature, policy and practice. Adelaide: Australian Centre for Child Protection, University of South Australia.

Hill EE, Thompson BJ, Williams EN (1997) A guide to conducting consensual qualitative research. Counseling Psychologist, 25(4), 517-72.

Keys D (2009) Children and homelessness: literature review. Melbourne Citymission, Research and Social Policy Unit. Melbourne: Salvation Army Australia Southern Territory.

Le Roux MC, Kemp R (2009) Effect of a companion dog on depression and anxiety levels of elderly residents in a long-term care facility. Japanese Psychogeriatric Society, 9(1), 23-26.

Lee S (2008) Homeless - non English speaking background women and children who are vctims of domestic violence. Discussion paper. Sydney: Immigrant Women's Speakout Association NSW.

McNicholas J, Collis G (2000) Dogs as catalysts for social interactions: robustness of the effect. British Journal of Psychology, 91(Pt 1), 61-70.

Noble-Carr D (2006) The experiences and effects of family homelessness for children. Literature review. Canberra: Institute of Child Protection Studies, Australian Catholic University.

O'Haire M (2009) The benefits of companion animals for human mental and physical health. 2009 RSPCA Australia Scientific Seminar: Animals and Human Health. Brisbane: Centre for Companion Animal Health, School of Veterinary Science, University of Queensland, 9.

Phillips Cohen S (2002) Can pets function as family members? Western Journal of Nursing Research, 24(6), 621-38.
Rew L (2000) Friends and pets as companions: strategies for coping with loneliness among homeless youth. Journal of Child and Adolescent Psychiatric Nursing, 13(3), 125-32.

Shore ER, Peterson CL, Douglas DK (2003) Moving as a reason for pet relinquishment: a closer look. Journal of Applied Animal Welfare Science, $6(1), 39-52$

Talbot C (2003) Social exclusion and homelessness: everyone's responsibility [online]. Adelaide: Uniting Care Wesley. Available at: http://www.ucwadel.org.au/publications/resources/Social_ Exclusion_Homelessness_Oct2003.pdf Accessed 2005. Cited in: St Luke's Anglicare (2005) No home, no kids: the vicious cycle of homelessness and out-of-home care placements, for families in Central Victoria. Bendigo: St Luke's Anglicare. Cited in: D Noble-Carr (2006) The experiences and effects of family homelessness for children. Literature review. Canberra: Institute of Child Protection Studies, Australian Catholic University.

Velde BP, Cipriani J, Grace F (2005) Resident and therapist views of animal assisted therapy: implications for occupational therapy practice. Australian Occupational Therapy Journal, 52(1), 43-50.

Virués-Ortega J, Buela-Casal G (2006) Psychophysiological effects of humananimal interaction: theoretical issues and long-term interaction effects. Journal of Nervous and Mental Disease, 194(1), 52-57.

Walsh $F$ (2009) Human-animal bonds 1: the relational significance of companion animals. Family Process, 48(4), 462-80.

Wood L (2009) Living well together: how companion animals can help strengthen social fabric. Perth: Centre for the Built Environment and Health (School of Population Health), University of Western Australia, and Petcare Information and Advisory Service Pty Ltd.

Zimalag U, Krupa T (2009) Pet ownership as a meaningful community occupation for people with serious mental illness. American Journal of Occupational Therapy, 63(2), 126-37. 